\title{
MODELAGEM E SIMULAÇÃO CFD DE TROCADORES DE CALOR COM ALETAS MODIFICADAS
}

\section{MODELING AND SIMULATION CFD OF COMPACTS HEAT EXCHANGERS WITH MODIFICATED FINS}

\author{
M. G. SABINO ${ }^{1, *}$, A. M. B. TIBIRIÇÁ ${ }^{1}$
}

${ }^{1}$ Federal University of Viçosa, Department of Mechanical Engineering, Viçosa, Minas Gerais, Brazil

${ }^{*}$ Millena Gomes Sabino. Federal University of Viçosa, Department of Mechanical Engineering, Viçosa, Minas Gerais, Brazil, Phone: +55 31986751994 e-mail address: millenagomes.sabino@gmail.com (M. G. SABINO).

\begin{tabular}{|c|}
\hline A R T I CLE INFO \\
\hline $\begin{array}{l}\text { Article history: } \\
\text { Received: 2017-09-22 } \\
\text { Accepted: 2018-03-22 } \\
\text { Available online: } 2018-06-30\end{array}$ \\
\hline $\begin{array}{l}\text { palavras-chave } \\
\text { Trocador de calor } \\
\text { Aletas modificadas } \\
\text { Fator j de Colburn } \\
\text { Fator f de atrito } \\
\text { Queda de pressão }\end{array}$ \\
\hline $\begin{array}{l}\text { keywords } \\
\text { Heat exchangers } \\
\text { Modified fins } \\
\text { Colburn j fator } \\
\text { Friction f fator } \\
\text { Pressure drop }\end{array}$ \\
\hline
\end{tabular}

\begin{abstract}
A B S T R A C T
Within the range of heat exchangers fin-tube type, the purpose of the present study was perform geometric alterations in a plane fin previously studied. The alterations consisted in the addition of some structures (louvers, Winglets and wavy geometry) that would increase the heat exchange. The execution of tasks was possible using the Numeric Simulation software called ANSYS for CFD. The results achieved were useful to analyze the dimensionless factors Colburn factor " $j$ " and Friction factor " $f$. The goal of this procedure was observe the heat exchanges and drop pressure variations. It was concluded that the geometric alterations effectively interfered in the heat exchange and pressure drop.
\end{abstract}

\section{R E S U M O / R E S U M E N}

Dentro do escopo de trocadores de calor do tipo tubo aleta, a proposta deste presente trabalho foi promover alterações geométricas em uma aleta plana previamente estudada. As alterações propostas consistiram no acréscimo de estruturas (Louvers, winglets e ondulações) que possibilitariam no aumento da troca de calor. A realização do estudo foi feita com o auxílio do software de simulação numérica ANSYS para CFD. Os resultados obtidos nas simulações foram úteis para a análise dos fatores adimensionais " $j$ ” de Colburn e " $f$ " de atrito com o intuído de observar as variações na troca de calor e queda de pressão nas aletas modificadas. Observou-se que as alterações geométricas, de fato, interferiram nos valores de troca de calor e queda de pressão. 


\section{INTRODUÇÃO}

Trocadores de calor são dispositivos que proporcionam troca de calor de maneira facilitada entre dois fluidos com temperatura diferentes, evitando a mistura entre os dois fluidos. Estes aparelhos são largamente utilizados para diferentes tipos de aplicações: Sistemas de aquecimento doméstico, instalações para condicionar o ar e processos químicos, por exemplo.

Em geral, as velocidades dos fluidos em trocadores variam entre 0,7 e $7 \mathrm{~m} / \mathrm{s}$ para líquidos e 3 e $30 \mathrm{~m} / \mathrm{s}$ para gases (Çengel, 2012). O uso de baixas velocidades é necessário para que não haja erosões, vibrações nos tubos, ruídos e principalmente perda de pressão. $\mathrm{O}$ aumento da transferência de calor em trocadores de calor é comumente acompanhado por um acréscimo na queda de pressão. Diante disso, a melhora na troca de calor deve ser avaliada em relação a queda de pressão.

No presente trabalho, trataremos de realizar alterações em trocadores de calor do tipo tubo-aleta, adicionando estruturas que exercem influência na troca de calor e queda de pressão do trocador O objetivo principal é observar se os resultados obtidos condizem com os resultados esperados de acordo com a teoria de Trocadores de Calor.

\subsection{Modificação em aletas}

O uso de aletas modificadas é a maneira mais eficaz de melhorar a troca de calor em um trocador de calor, atendendo, desse modo, as exigências de alta eficiência com baixo custo.

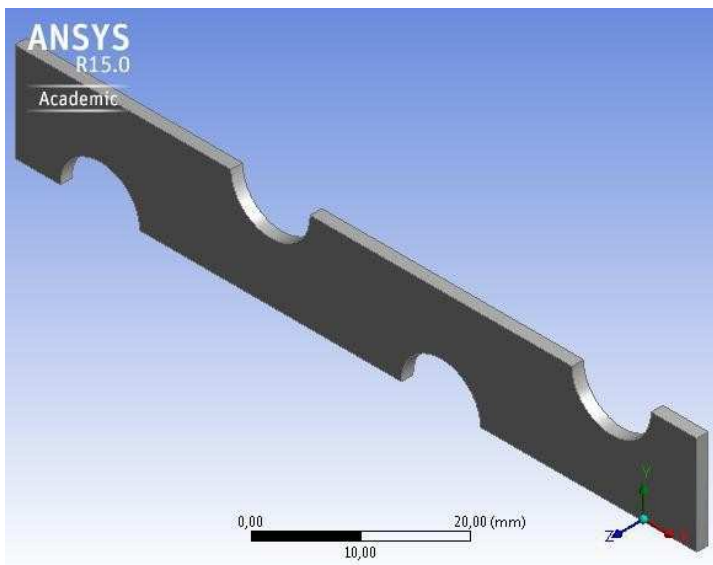

Figura 1 - Geometria de aleta plana utilizada no trabalho.

As aletas modificadas são capazes de aumentar a superfície de troca de calor e alterar o fluxo convencional do ar. Normalmente, usam-se superfícies onduladas e acréscimo de louvers para essa finalidade. A superfície ondulada é capaz de mudar periodicamente a direção do fluxo principal e provocar melhor mistura do fluido, louvers podem "interromper" periodicamente o fluxo principal e renovar constantemente a camada limite térmica (Tian, et al 2009). As modificações realizadas nessa geometria base consistiram no acréscimo de Louvers, Winglets $e$ ondulações na aleta plana mostrada na figura 1.

\subsection{Características do escoamento}

O escoamento em estudo é caracterizado como convecção forçada interna, normalmente usado em aplicações de aquecimento e resfriamento. Em escoamentos internos o fluido está confinado por superfícies e, portanto, existe um limite sobre quanto a camada limite pode crescer. $O$ fluido, nesses casos, é forçado a fluir por meio de um ventilador ou bomba através de uma seção de escoamento suficientemente longa capaz de proporcionar a troca de calor necessária.

\subsection{Adimensionais}

O número de Reynolds relaciona forças de inércia com forças viscosas e quanto maior o número de Reynolds, maior serão as forças de inércia que caracterizarão o regime de escoamento.

$$
R e=\frac{\rho V D_{C}}{\mu}
$$

A queda de pressão está representada pelo adimensional fator de atrito (equação 2) que leva em consideração a tensão de cisalhamento na parede (equação 3) que é calculado em relação à velocidade de entrada, dimensões da geometria e propriedades do fluido. Além disso, deve-se levar em consideração que para convecção forçada a queda de pressão em relação à tensão de cisalhamento na parede é dada pela equação 4 .

$$
\begin{aligned}
& f=\frac{\Delta P}{\rho V^{2}} \frac{D_{c}}{2 L} \\
& \tau=f \frac{\rho V^{2}}{2} \\
& \Delta P=\frac{4 L}{D_{c}} \tau
\end{aligned}
$$

E a taxa de transferência de calor será representada pelo adimensional fator j de Colburn (equação 5) que além de ser em função do adimensional de Reynolds, é função de outros dois adimensionais: $\mathrm{O}$ adimensional Nusselt (equação 6) que evidencia o quanto a transferência de calor ocorre por convecção; e o adimensional Prandtl (Pr) que relaciona propriedades do fluido que nos diz respeito à camadas limite térmica e hidrodinâmica. Para $\operatorname{Pr}<1$, significa que o calor se difunde mais rapidamente e para $\operatorname{Pr}>1$, o contrário.

$$
\begin{aligned}
& j=\frac{N u}{\operatorname{RePr}^{\frac{1}{3}}} \\
& N u=\frac{h D_{C}}{k}
\end{aligned}
$$

\section{MATERIAIS E MÉTODOS}

O estudo de aletas planas com tubos intercalados foi realizado previamente e possibilitou, portanto, o desenvolvimento do presente trabalho. Na ocasião de estudo de aletas planas foi validado um modelo computacional com base em dados experimentais obtidos por Wang et al (1996) e comparou com os resultados com o modelo CFD de Bhuiyan et al (2013). Este trabalho precedente serviu, portanto, para a criação de um modelo computacional baseado em dados experimentais e foi de suma importância para o desenvolvimento do trabalho atual.

\subsection{Considerações no modelo CFD}

O fluido que escoa em um trocador de calor aplicado em ar condicionado é o ar. As seguintes propriedades serão consideradas constantes: $\mathrm{k}=0.0261 \mathrm{~W} / \mathrm{m} \mathrm{K}, \mu=1.831 \times 10-5 \mathrm{Ns} / \mathrm{m}^{2}, \operatorname{Pr}=0.736$, $\rho=1.185 \mathrm{kgm}-3$. O fluido também será tratado como fluido incompressível.

\subsection{Modelo matemático}

As equações governantes do fenômeno em estudo, incluem as equações de conservação da massa e momento, equação de energia e as equações características do modelo de turbulência adotado (k-ômega). A resolução dessas equações é impraticável de 
forma analítica e, portanto, utiliza-se ferramentas o CFD para a resolução numérica iterativa.

\subsection{Modelo computacional}

Para a realização de uma simulação CFD primeiramente deve-se criar uma geometria tridimensional que reproduza as dimensões do fluido em análise. Posteriormente, deve-se criar a malha. Essa estrutura subdivide o domínio total em volumes de controle menores, onde as equações governantes são resolvidas para cada um desses volumes de controle. Por fim, segue-se a configuração das condições de contorno do problema e simulação do mesmo.

A etapa de criação da malha é de extrema importância para a qualidade e confiabilidade dos resultados obtidos, pois determina as regiões do domínio em que os volumes de controle devem ser menores.

Geometrias: A geometria base apresenta as seguintes especificações: $\mathrm{D}=10,23 \mathrm{~mm}, \mathrm{Fp}=3 \mathrm{~mm}, \mathrm{Lt}=25,4 \mathrm{~mm}, \mathrm{Ll}=22$ $\mathrm{mm}$ e $\mathrm{N}=4$. Considerando que nos tubos e aletas, a temperatura não se altera, não foi necessário acrescentar a parte sólida dos mesmos, apenas a parte fluida foi criada, ou seja, a parte onde o fluido ar escoa.
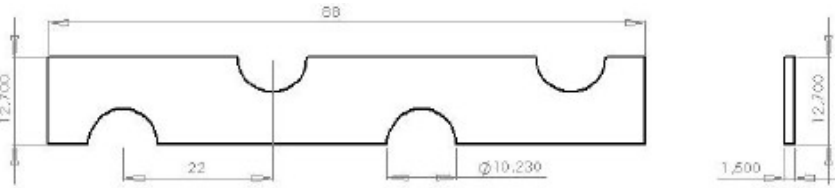

Figura 2 - Dimensões detalhadas da aleta plana.

Desse modo, as modificações nessa geometria base consistiram no acréscimo de Louvers, Winglets $e$ ondulações na aleta plana. Estas modificações estão mostradas nas figuras 3, 4 e 5 a seguir.

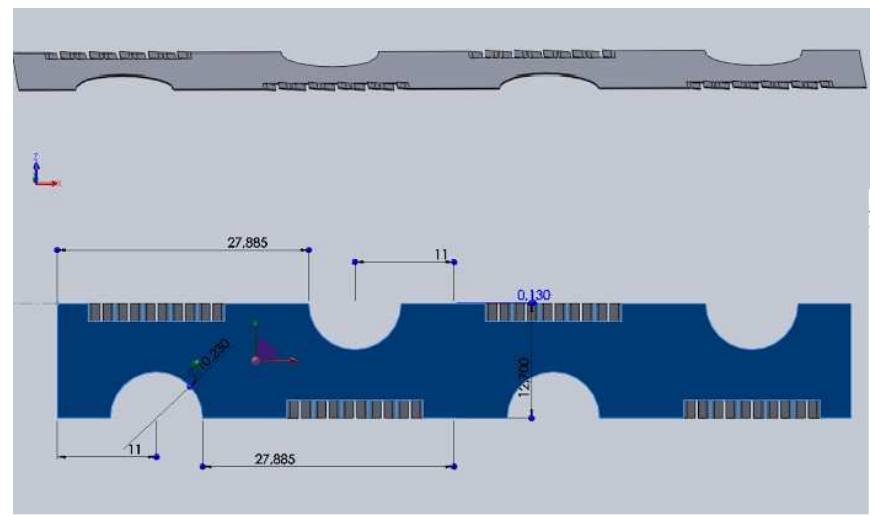

Figura 3 - Aleta com louvers.

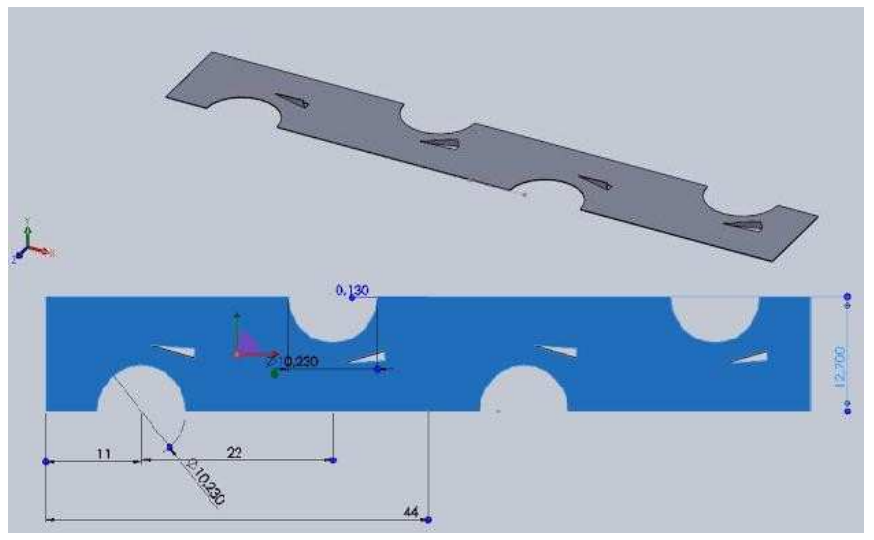

Figura 4 - Aleta com winglets.

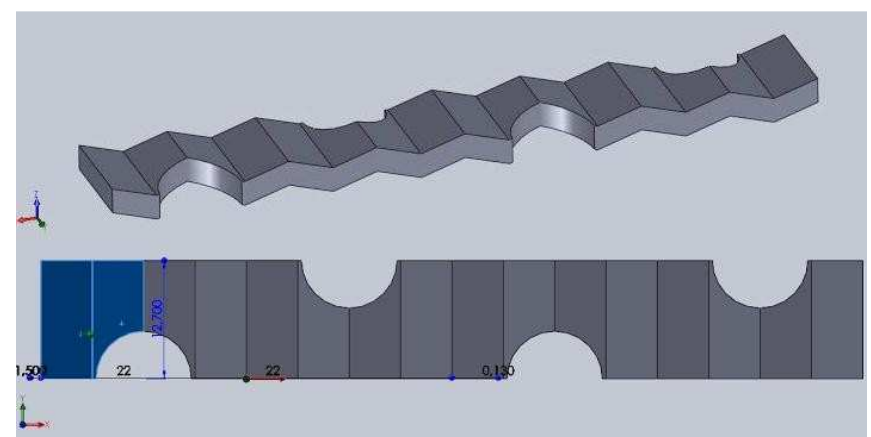

Figura 5 - Aleta com ondulações.

Padrão de construção de malhas: Foram construídas três tipos de malhas com configurações diferentes e cada tipo de malha foi aplicado nas três diferentes geometrias de aleta. $\mathrm{O}$ software utilizado nessa etapa foi o Meshing do pacote ANSYS(15.0). A malha 1 possui refinamento nas proximidades e curvaturas da geometria e ausência de Inflation .Os tamanhos mínimo e máximo de cada elemento são 0,250 e 0,300 mm, respectivamente. Na figura 6 abaixo, é possível ver em detalhes os elementos de malha gerados.
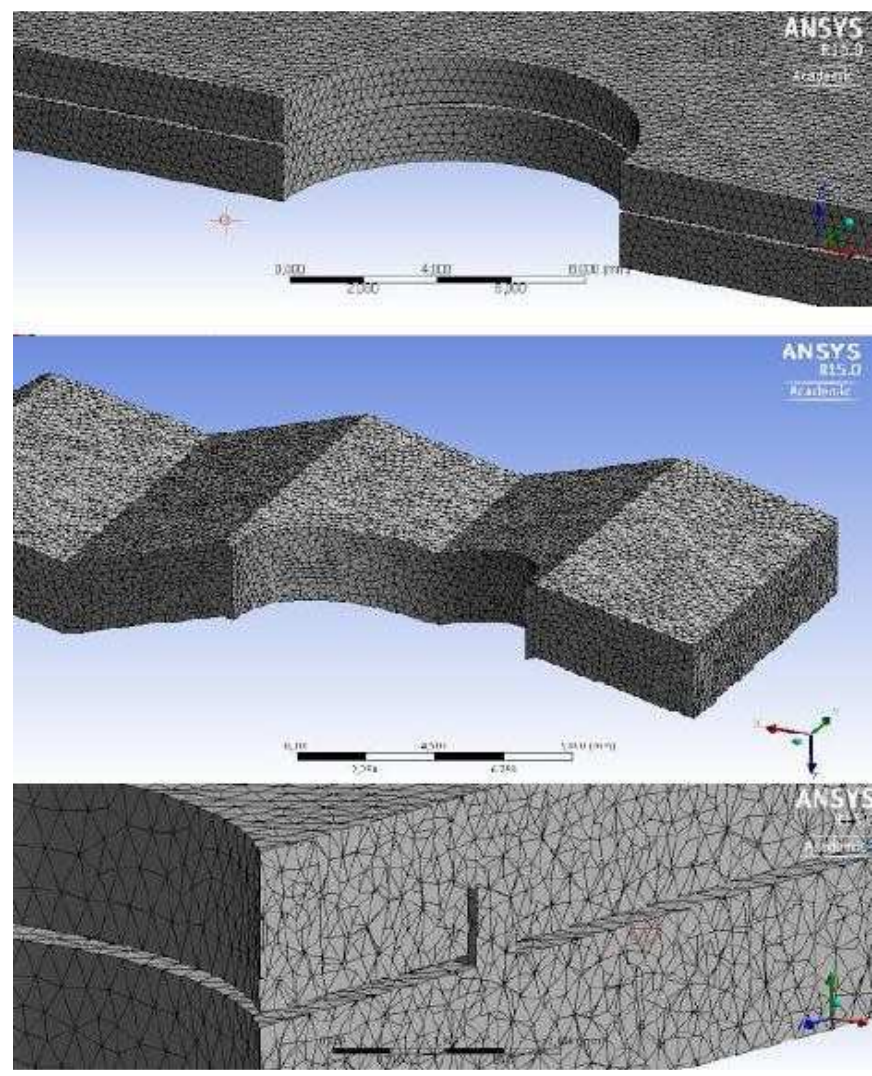

Figura 6 - Malha tipo 1 aplicada nas três geometrias.

A malha 2 possui refinamentos nas proximidades e curvaturas da geometria, tamanho mínimo e máximo da face dos elementos iguais a 4,4342 x $10^{\wedge}(-2)$ e 4,4342 mm, respectivamente. Já a malha 3 possui refinamento nas proximidades e curvaturas da geometria. Há presença de inflation: $\mathrm{O}$ tamanho do primeiro elemento é $0,038 \mathrm{~mm}$, taxa de crescimento dos elementos é de $10 \%$ e número máximo de camadas do inflation igual a 6.

Modelo de turbulência e Condições de contorno: $\mathrm{O}$ modelo de turbulência utilizado foi o k-omega. A formulação desse modelo é adequada para captar informações de domínios em que a camada limite tem grande influência nos resultados. As condições de contorno são as configurações escolhidas para 
o modelo CFD, utilizando-se do software $C F X$ da plataforma ANSYS. Nesse trabalho, as condições de contorno para cada geometria estão mostradas na figura 7 .

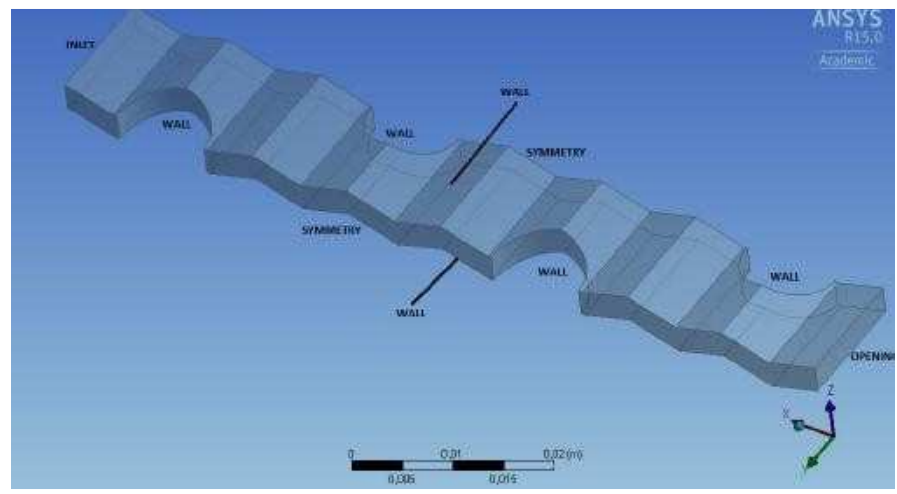

Figura 7 - Condições de contorno.

Método para adequação de malha: Após a construção e simulação das geometrias com as três malhas já descritas, a malha considerada como a de melhor qualidade e com parâmetros mais criteriosos foi a malha 3 . Isso se justifica devido a aproximação do valor de $\mathrm{y}+$ encontrado com o valor de $\mathrm{y}+$ ideal sugerido pelos tutoriais oficiais do software CFX.

A partir dos valores obtidos nas simulações para as cinco velocidades para as grandezas "transferência de calor nas paredes", "queda de pressão" e "temperatura média" foi possível calcular os valores dos adimensionais $\mathrm{j}$ e f. Esses adimensionais foram comparados aos valores encontrados nas simulações para aletas planas.

\section{RESULTADOS E DISCUSSÃO}

Os resultados obtidos de troca de calor(W), queda de pressão $(\mathrm{Pa})$ e temperatura média do domínio $\left({ }^{\circ} \mathrm{C}\right)$ foram úteis para o cálculo dos adimensionais: Fator de atrito "f", fator de de Colburn “j”. O valor do número de Reynolds variou de 1300 a 2000. As análises percentuais feitas a seguir foram baseadas no Reynolds igual a 1300 em que todas as aletas modificadas foram comparadas às aletas lisas. Também torna-se pertinente uma análise visual das simulações, observando a distribuição da transferência de calor, pressões e velocidades no domínio de simulação.

Imagens dos resultados - Pressão, Fluxo de calor e Velocidade: As figuras 8, 9 e 10 mostram o comportamento de pressão e fluxo de calor ao longo das aletas com louvers, winglets e ondulações, respectivamente. As figuras 11, 12 e 13 mostram o desenvolvimento da velocidade ao longo das aletas para geometrias com louvers, winglets e ondulações, respectivamente. Análises e comentários serão feitos após a figura 13 .

Observando as figuras relativas às aletas com louvers, vê-se que houve queda de pressão do início do domínio para o final e que na região onde existem os louvers o fluxo de calor teve valores maiores que no resto da aleta. Quanto à velocidade, esta assume seus maiores valores nas bordas laterais dos tubos. Isso se justifica devido à uma região de baixa pressão existente.

$\mathrm{Na}$ geometria onde há acréscimo de winglets ocorre visível queda de pressão do início do domínio para o final e a velocidade também assume seus maiores valores na borda lateral dos tubos. Quanto ao fluxo de calor, ocorre pequeno aumento na transferência de calor na região dos winglets.

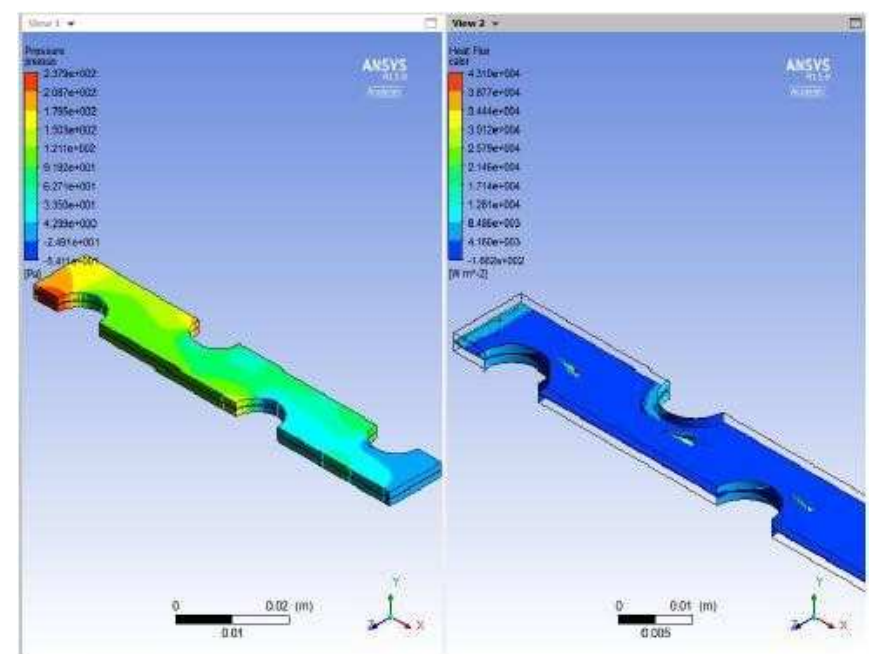

Figura 8 - Resultados gráficos de pressão e fluxo de calor para aleta com louvers.

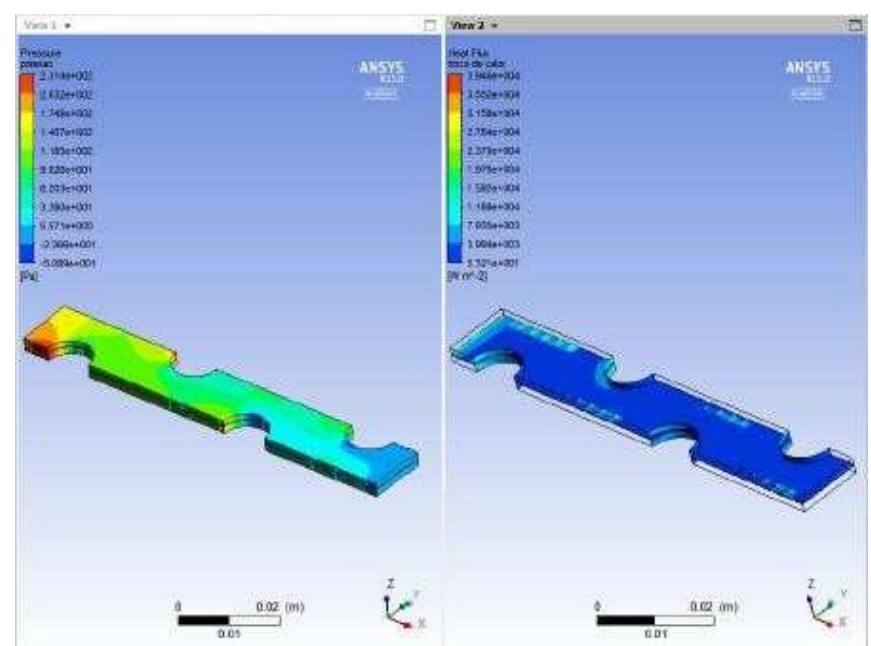

Figura 9 - Resultados gráficos de pressão e fluxo de calor para aletas com winglets

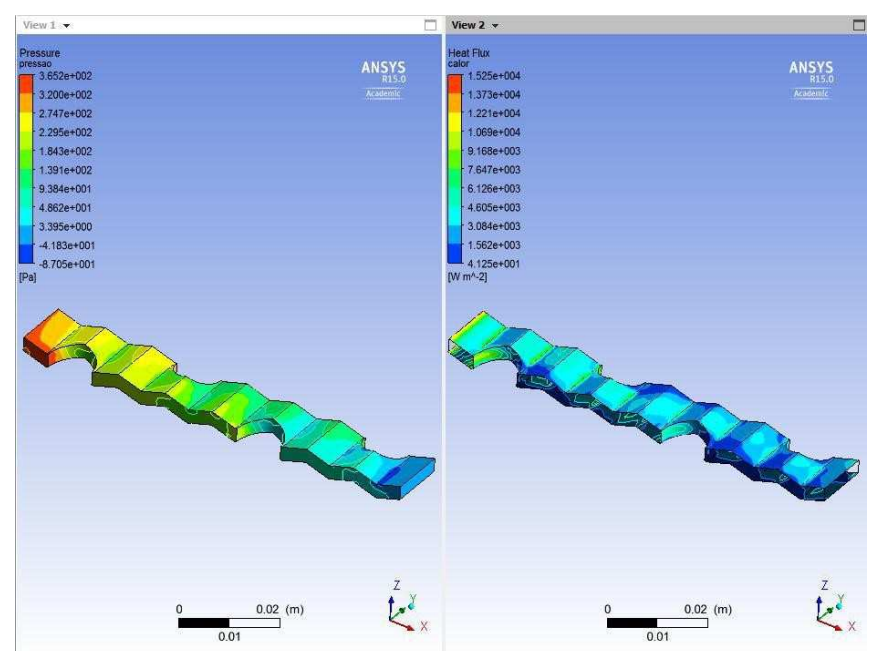

Figura 10 - Resultados gráficos de pressão e fluxo de calor pala aletas onduladas. 


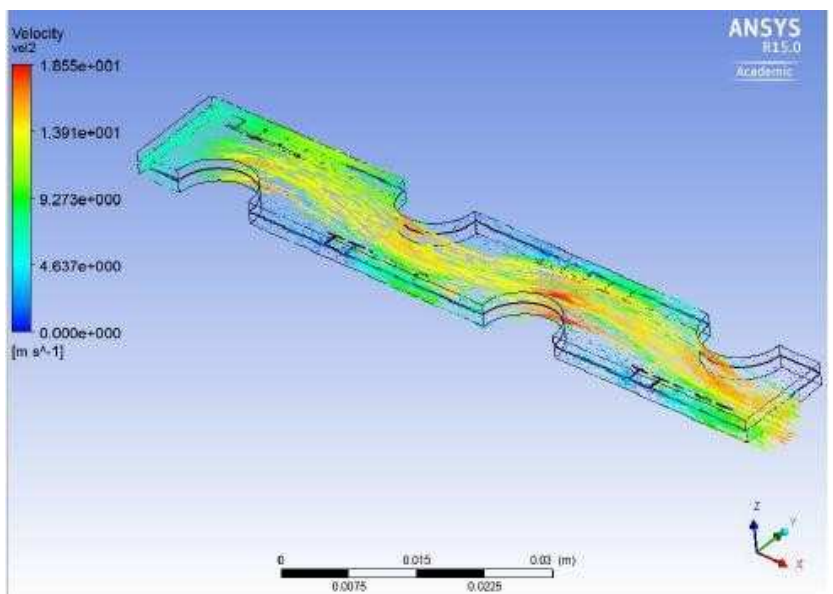

Figura 11 - Velocidade ao longo de aleta com louvers.

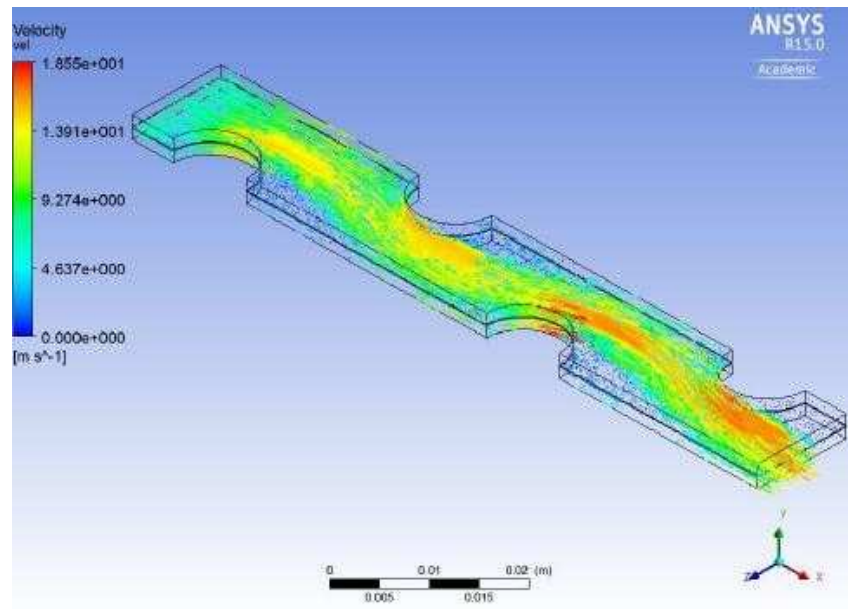

Figura 12 - Velocidade ao longo de aleta com winglets.

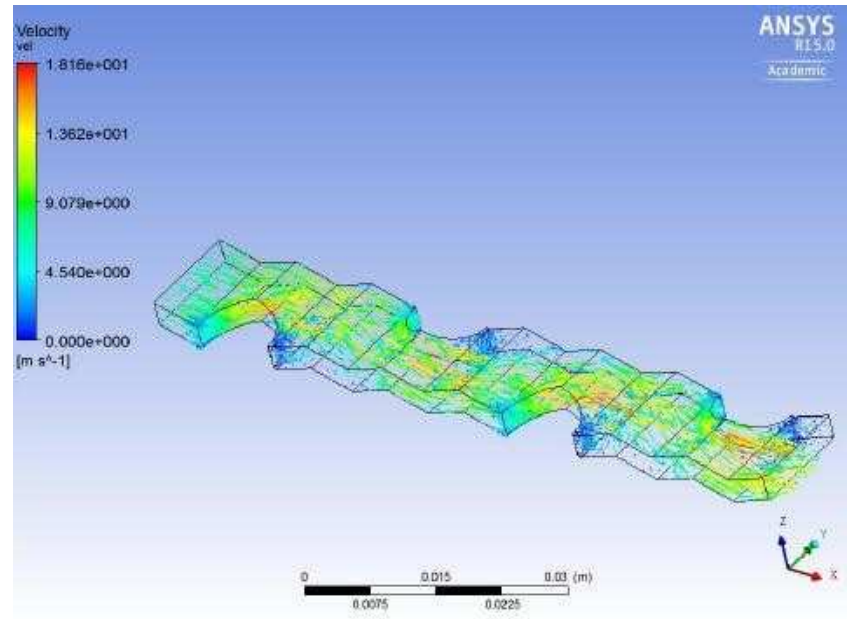

Figura 13 - Velocidade ao longo de aleta ondulada.

$\mathrm{Na}$ geometria com ondulações, ocorre nítida queda de pressão ao longo do domínio de simulação e a troca de calor assume valores máximos nas superfícies das aletas. As maiores velocidades, assim como nas outras geometrias, também são encontradas nas laterais dos tubos.

Análise dos resultados - Fatores adimensionais "j" e "f": Observa-se que o fator $\mathrm{j}$ teve aumento de $10,9 \%, 12,4 \%$ e $43,0 \%$ para aletas com winglets, louvers e ondulações, respectivamente. Já o fator j teve acréscimo de 91,92\%, 15,9\% e 12,3\% para aletas com ondulações, winglets e louvers, respectivamente.
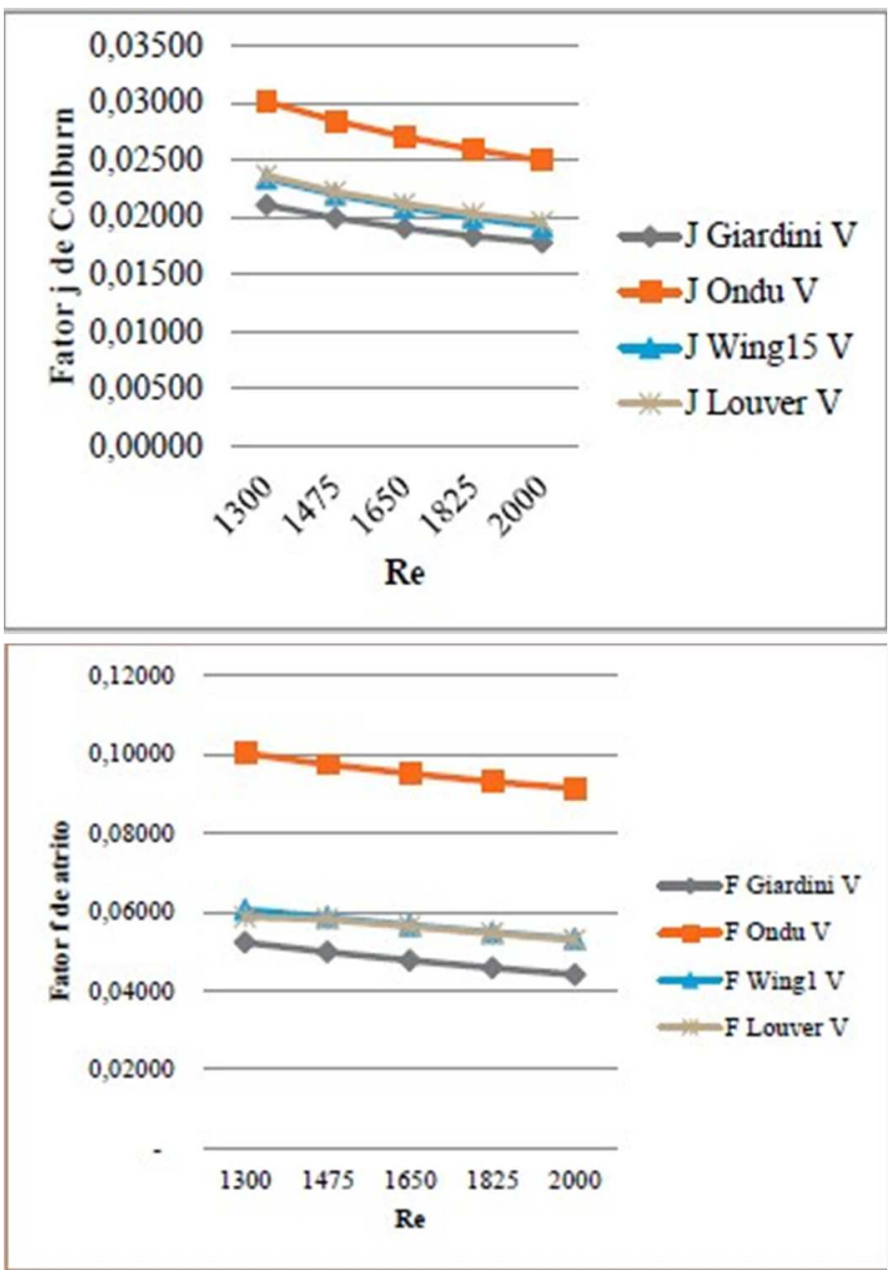

Figura 14 - Gráficos de fatores "j" e "f" para malha tipo 3 (Considerada malha mais adequada).

\section{CONCLUSÃO}

É possível concluir depois deste trabalho que os resultados obtidos estão compatíveis com os resultados esperados, pois as modificações nas aletas propiciaram aumento do fluxo de calor e, em alguns casos, aumento da queda de pressão. Além disso, como conclusão secundária é possível observar que as simulações feitas com três diferentes tipos de malhas evidenciaram a importância da confecção de uma malha adequada, para cada geometria estudada.

O desenvolvimento deste trabalho contribuiu enormemente com o desenvolvimento da área de trocadores de calor no NEMOS e também se mostrou como um direcionamento para estudos posteriores no assunto.

\section{R E F E R E N C I A S}

AMEEL, B.; HUISSEUNE, H.; DEGROOTE, J.; T'JOEN, C.; JAEGER, P.; VIERENDEELS, J.; PAEPE, M. On fin efficiency in interrupted fin and tube heat exchangers. International Journal of Heat and Mass Transfer 2013.

BHUIYAN, A. A.; AMIN, M, R.; ISLAM, A. K. M. S. Threedimensional performance analysis of plain fin tube heat exchangers in transitional regime. Applied Thermal Engineering. 2013.

CARIJA, Z.; FRANCOVIC, B.; PERCIC, M.; CAVRAK, M. Heat transfer analysis of fin-and-tube heat exchangers 
with flat and louvered fin geometries. International Journal of Refrigeration 2014.

ÇENGEL, Y. A.; GHAJAR, A. J. Transferência de calor e massa- Uma abordagem prática. São Paulo: McGrawHill do Brasil, 2012.

MARTINS RIBEIRO GIARDINI, L., MESSIAS BINHONHA TIBIRIÇÁ, MODELAGEM E SIMULAÇÃO CFD DE TROCADOR DE CALOR PARA CONDICIONAMENTO DE AR. The Journal of Engineering and Exact Sciences, Viçosa, 2, nov. 2016. Disponível em: http://www.seer.ufv.br/seer/rbeq2/index.php/req2/article /view/140/166. Acesso em: 05 agosto 2017.

TIAN, L.; HE, Y.; TAO, Y.; TAO W. A comparative study on the air-side performance of wavy fin- and-tube heat exchanger with punched delta winglets in staggered and in-line arrangements. International Journal of Thermal Sciences 2009

WANG, C.; CHANG, Y.; HSIEH Y.; LIN, Y. Sensible heat and friction characteristics of plate fin- and-tube heat exchangers having plane fins. International Journal of Refrigeration, 1996.

HUISSEUNE, H.; T'JOEN, C.; JAEGER, P.; AMEEL B.; SCHAMPHELEIR, S.; PAEPE, M. Performance enhancement of a louvered fin heat exchanger by using delta winglet vortex generators. International Journal of Heat and Mass Transfer, 2013. 